\title{
A certain class of completely monotonic sequences
}

Senlin Guo ${ }^{1 *}$, Hari M Srivastava² and Necdet Batir ${ }^{3}$

${ }^{\text {"Correspondence: }}$ sguo@hotmail.com

1 Department of Mathematics, Zhongyuan University of

Technology, Zhengzhou, Henan 450007, People's Republic of China Full list of author information is available at the end of the article

\begin{abstract}
In this article, we present some necessary conditions, a sufficient condition and a necessary and sufficient condition for sequences to be completely monotonic. One counterexample is also presented.

MSC: Primary 40A05; secondary 26A45; 26A48; 39A60

Keywords: necessary condition; sufficient condition; necessary and sufficient condition; difference equation; moment sequence; completely monotonic sequence; completely monotonic function; bounded variation; Stieltjes integral
\end{abstract}

\section{Introduction and the main results}

We first recall some definitions and basic results on or related to completely monotonic sequences and completely monotonic functions.

Definition 1 [1] A sequence $\left\{\mu_{n}\right\}_{n=0}^{\infty}$ is called a moment sequence if there exists a function $\alpha(t)$ of bounded variation on the interval $[0,1]$ such that

$$
\mu_{n}=\int_{0}^{1} t^{n} d \alpha(t), \quad n \in \mathbb{N}_{0}
$$

Here, in Definition 1 and throughout the paper,

$$
\mathbb{N}_{0}:=\{0\} \cup \mathbb{N},
$$

and $\mathbb{N}$ is the set of all positive integers.

Definition $2[1]$ A sequence $\left\{\mu_{n}\right\}_{n=0}^{\infty}$ is called completely monotonic if

$$
(-1)^{k} \Delta^{k} \mu_{n} \geqq 0, \quad n, k \in \mathbb{N}_{0},
$$

where

$$
\Delta^{0} \mu_{n}=\mu_{n}
$$

and

$$
\Delta^{k+1} \mu_{n}=\Delta^{k} \mu_{n+1}-\Delta^{k} \mu_{n} .
$$

\section{囪 Springer}

○2013 Guo et al.; licensee Springer. This is an Open Access article distributed under the terms of the Creative Commons Attribution License (http://creativecommons.org/licenses/by/2.0), which permits unrestricted use, distribution, and reproduction in any medium, provided the original work is properly cited. 
Such a sequence is called totally monotone in [2].

From Definition 2, using mathematical induction, we can prove, for a completely monotonic sequence $\left\{\mu_{n}\right\}_{n=0}^{\infty}$, that the sequence $\left\{(-1)^{m} \Delta^{m} \mu_{n}\right\}_{n=0}^{\infty}$ is non-increasing for any fixed $m \in \mathbb{N}_{0}$, and that the sequence $\left\{(-1)^{m} \Delta^{m} \mu_{n}\right\}_{m=0}^{\infty}$ is non-increasing for any fixed $n \in \mathbb{N}_{0}$. The difference equation (4) plays an important role in the proofs of these properties and our main results of this paper.

In [3], the authors showed that for a completely monotonic sequence $\left\{\mu_{n}\right\}_{n=0}^{\infty}$, we always have

$$
(-1)^{k} \Delta^{k} \mu_{n}>0, \quad n, k \in \mathbb{N}_{0},
$$

unless $\mu_{n}=c$, a constant for all $n \in \mathbb{N}$.

Let

$$
\lambda_{k, m}:=\left(\begin{array}{c}
k \\
m
\end{array}\right)(-1)^{k-m} \Delta^{k-m} \mu_{m}, \quad k, m \in \mathbb{N}_{0} .
$$

It was shown (see [1]) as follows.

Theorem 1 A sequence $\left\{\mu_{n}\right\}_{n=0}^{\infty}$ is a moment sequence if and only if there exists a constant L such that

$$
\sum_{m=0}^{k}\left|\lambda_{k, m}\right|<L, \quad k \in \mathbb{N}_{0}
$$

where in (7), $\lambda_{k, m}$ is defined by (6).

For completely monotonic sequences, the following is the well-known Hausdorff's theorem (see [1]).

Theorem 2 A sequence $\left\{\mu_{n}\right\}_{n=0}^{\infty}$ is completely monotonic if and only if there exists a nondecreasing and bounded function $\alpha(t)$ on $[0,1]$ such that

$$
\mu_{n}=\int_{0}^{1} t^{n} d \alpha(t), \quad n \in \mathbb{N}_{0}
$$

From this theorem, we know (see [1]) that a completely monotonic sequence is a moment sequence and is as follows.

Theorem 3 A necessary and sufficient condition that the sequence $\left\{\mu_{n}\right\}_{n=0}^{\infty}$ should be a moment sequence is that it should be the difference of two completely monotonic sequences.

We also recall the following definition.

Definition 3 [1] A function $f$ is said to be completely monotonic on an interval $I$ if $f$ is continuous on $I$ has derivatives of all orders on $I^{o}$ (the interior of $I$ ) and for all $n \in \mathbb{N}_{0}$,

$$
(-1)^{n} f^{(n)}(x) \geqq 0, \quad x \in I^{o} .
$$


Some mathematicians use the terminology completely monotone instead of completely monotonic. The class of all completely monotonic functions on the interval $I$ is denoted by $\mathrm{CM}(I)$.

The completely monotonic functions and completely monotonic sequences have remarkable applications in probability and statistics [4-10], physics [11,12], numerical and asymptotic analysis [2], etc.

For the completely monotonic functions on the interval $[0, \infty)$, Widder proved (see [1]).

Theorem 4 A function $f$ on the interval $[0, \infty)$ is completely monotonic if and only if there exists a bounded and non-decreasing function $\alpha(t)$ on $[0, \infty)$ such that

$$
f(x)=\int_{0}^{\infty} e^{-x t} d \alpha(t)
$$

There is rich literature on completely monotonic functions. For more recent works, see, for example, [13-26].

There exists a close relationship between completely monotonic functions and completely monotonic sequences. For example, Widder [27] showed the following.

Theorem 5 Suppose that $f \in \mathrm{CM}[a, \infty)$, then for any $\delta \geqq 0$, the sequence $\{f(a+n \delta)\}_{n=0}^{\infty}$ is completely monotonic.

This result was generalized in [28] as follows.

Theorem 6 Suppose that $f \in \mathrm{CM}[a, \infty)$. If the sequence $\left\{\Delta x_{k}\right\}_{k=0}^{\infty}$ is completely monotonic and $x_{0} \geqq a$, then the sequence $\left\{f\left(x_{k}\right)\right\}_{k=0}^{\infty}$ is also completely monotonic.

For the meaning of $\Delta x_{k}, k \in \mathbb{N}_{0}$ in Theorem 6, see (3) and (4).

Suppose that $f \in \operatorname{CM}[0, \infty)$. By Theorem 5 , we know that $\{f(n)\}_{n=0}^{\infty}$ is completely monotonic.

The following result was obtained in [16].

Theorem 7 Suppose that the sequence $\left\{\mu_{n}\right\}_{n=0}^{\infty}$ is completely monotonic, then for any $\varepsilon \in$ $(0,1)$, there exists a continuous interpolating function $f(x)$ on the interval $[0, \infty)$ such that $\left.f\right|_{[0, \varepsilon]}$ and $\left.f\right|_{[\varepsilon, \infty)}$ are both completely monotonic and

$$
f(n)=\mu_{n}, \quad n \in \mathbb{N}_{0} .
$$

From this result or Theorem 2, we can get the following.

Theorem 8 Suppose that the sequence $\left\{\mu_{n}\right\}_{n=0}^{\infty}$ is completely monotonic. Then there exists a completely monotonic interpolating function $g(x)$ on the interval $[1, \infty)$ such that

$$
g(n)=\mu_{n}, \quad n \in \mathbb{N} .
$$

It should be noted that (see [1, Chapter IV]) under the condition of Theorem 8, we cannot guarantee that there exists a completely monotonic interpolating function $g(x)$ on the 
interval $[0, \infty)$ such that

$$
g(n)=\mu_{n}, \quad n \in \mathbb{N}_{0} .
$$

In this article, we shall further investigate the properties of the completely monotonic sequences. We shall give some necessary conditions, a sufficient condition and a necessary and sufficient condition for sequences to be completely monotonic. More precisely we have the following results.

Theorem 9 Suppose that the sequence $\left\{\mu_{n}\right\}_{n=0}^{\infty}$ is completely monotonic. Then, for any $m \in$ $\mathbb{N}_{0}$, the series

$$
\sum_{j=0}^{\infty}(-1)^{j} \Delta^{j} \mu_{m+1}
$$

converges and

$$
\mu_{m} \geqq \sum_{j=0}^{\infty}(-1)^{j} \Delta^{j} \mu_{m+1} .
$$

Corollary 1 Suppose that the sequence $\left\{\mu_{n}\right\}_{n=0}^{\infty}$ is completely monotonic. Then for $m, k \in$ $\mathbb{N}_{0}$,

$$
\mu_{m}=(-1)^{k+1} \Delta^{k+1} \mu_{m}+\sum_{i=0}^{k}(-1)^{i} \Delta^{i} \mu_{m+1} .
$$

Remark 1 Although from the complete monotonicity of the sequence $\left\{\mu_{n}\right\}_{n=0}^{\infty}$, we can deduce that for any $m \in \mathbb{N}_{0}$, the series

$$
\sum_{j=0}^{\infty}(-1)^{j} \Delta^{j} \mu_{m+1}
$$

converges, it cannot guarantee the convergence of the series

$$
\sum_{j=0}^{\infty}(-1)^{j} \Delta^{j} \mu_{0}
$$

For example, let

$$
\mu_{n}=\frac{1}{n+1}, \quad n \in \mathbb{N}_{0} .
$$

Since the function

$$
f(x)=\frac{1}{x+1}
$$

is completely monotonic on the interval $[0, \infty)$, by Theorem 5 , we see that the sequence

$$
\left\{\mu_{n}\right\}_{n=0}^{\infty}:=\{f(n)\}_{n=0}^{\infty}=\left\{\frac{1}{n+1}\right\}_{n=0}^{\infty}
$$


is completely monotonic. This conclusion can also be obtained by setting

$$
\alpha(t)=t
$$

in Theorem 2.

We can verify that

$$
\Delta^{j} \mu_{0}=\frac{(-1)^{j}}{j+1}
$$

Hence,

$$
\sum_{j=0}^{\infty}(-1)^{j} \Delta^{j} \mu_{0}=\sum_{j=0}^{\infty} \frac{1}{j+1}
$$

is the famous harmonic series, which is divergent.

Theorem 10 Suppose that the sequence $\left\{\mu_{n}\right\}_{n=0}^{\infty}$ is completely monotonic. Then for any $k, m \in \mathbb{N}_{0}$,

$$
(-1)^{k} \Delta^{k} \mu_{m} \geqq \sum_{j=k}^{\infty}(-1)^{j} \Delta^{j} \mu_{m+1} .
$$

Theorem 11 Suppose that the sequence $\left\{\mu_{n}\right\}_{n=1}^{\infty}$ is completely monotonic and that the series

$$
\sum_{j=0}^{\infty}(-1)^{j} \Delta^{j} \mu_{1}
$$

converges. Let $\mu_{0}$ be such that

$$
\mu_{0} \geqq \sum_{j=0}^{\infty}(-1)^{j} \Delta^{j} \mu_{1}
$$

Then the sequence $\left\{\mu_{n}\right\}_{n=0}^{\infty}$ is completely monotonic.

Theorem 12 A necessary and sufficient condition for the sequence $\left\{\mu_{n}\right\}_{n=0}^{\infty}$ to be completely monotonic is that the sequence $\left\{\mu_{n}\right\}_{n=1}^{\infty}$ is completely monotonic, the series

$$
\sum_{j=0}^{\infty}(-1)^{j} \Delta^{j} \mu_{1}
$$

converges and

$$
\mu_{0} \geqq \sum_{j=0}^{\infty}(-1)^{j} \Delta^{j} \mu_{1} .
$$




\section{Proofs of the main results}

Now, we are in a position to prove the main results.

Proof of Theorem 9 Since $\left\{\mu_{n}\right\}_{n=0}^{\infty}$ is completely monotonic, by Theorem 2, there exists a non-decreasing and bounded function $\alpha(t)$ on the interval $[0,1]$ such that

$$
\mu_{n}=\int_{0}^{1} t^{n} d \alpha(t), \quad n \in \mathbb{N}_{0}
$$

From (3), (4) and (13), we can prove that

$$
(-1)^{i} \Delta^{i} \mu_{n}=\int_{0}^{1}(1-t)^{i} t^{n} d \alpha(t), \quad i, n \in \mathbb{N}_{0} .
$$

Now, for $k \in \mathbb{N}$, we have

$$
\begin{aligned}
\sum_{i=0}^{k-1}(-1)^{i} \Delta^{i} \mu_{m+1} & =\sum_{i=0}^{k-1} \int_{0}^{1}(1-t)^{i} t^{m+1} d \alpha(t) \\
& =\int_{0}^{1} t^{m+1} \sum_{i=0}^{k-1}(1-t)^{i} d \alpha(t) \\
& =\int_{0}^{1} t^{m}\left(1-(1-t)^{k}\right) d \alpha(t) \\
& =\int_{0}^{1} t^{m} d \alpha(t)-\int_{0}^{1}(1-t)^{k} t^{m} d \alpha(t) \\
& =\mu_{m}-(-1)^{k} \Delta^{k} \mu_{m}, \quad m \in \mathbb{N}_{0} .
\end{aligned}
$$

Hence, for $k \in \mathbb{N}$,

$$
\mu_{m}=(-1)^{k} \Delta^{k} \mu_{m}+\sum_{i=0}^{k-1}(-1)^{i} \Delta^{i} \mu_{m+1}, \quad m \in \mathbb{N}_{0} .
$$

Since

$$
(-1)^{i} \Delta^{i} \mu_{n} \geqq 0, \quad i, n \in \mathbb{N}_{0},
$$

from (15), we get, for $k \geqq 1$,

$$
\mu_{m} \geqq \sum_{i=0}^{k-1}(-1)^{i} \Delta^{i} \mu_{m+1}, \quad m \in \mathbb{N}_{0} .
$$

From (16), we also know that

$$
\sum_{j=0}^{\infty}(-1)^{j} \Delta^{j} \mu_{m+1}, \quad m \in \mathbb{N}_{0}
$$


is a positive series. Then by (17), we obtain that

$$
\sum_{j=0}^{\infty}(-1)^{j} \Delta^{j} \mu_{m+1}, \quad m \in \mathbb{N}_{0}
$$

converges and that

$$
\mu_{m} \geqq \sum_{j=0}^{\infty}(-1)^{j} \Delta^{j} \mu_{m+1}, \quad m \in \mathbb{N}_{0} .
$$

The proof of Theorem 9 is thus completed.

Proof of Corollary 1 This corollary can be obtained from (15).

Proof of Theorem 10 Let $m$ be a fixed non-negative integer.

From Theorem 9, we see that

$$
\mu_{m} \geqq \sum_{j=0}^{\infty}(-1)^{j} \Delta^{j} \mu_{m+1},
$$

which means that (12) is valid for $k=0$.

Suppose that (12) is valid for $k=r$. Then

$$
\begin{aligned}
(-1)^{r+1} \Delta^{r+1} \mu_{m} & =(-1)^{r+1}\left(\Delta^{r} \mu_{m+1}-\Delta^{r} \mu_{m}\right) \\
& =(-1)^{r}\left(\Delta^{r} \mu_{m}-\Delta^{r} \mu_{m+1}\right) \\
& =(-1)^{r} \Delta^{r} \mu_{m}-(-1)^{r} \Delta^{r} \mu_{m+1} \\
& \geqq \sum_{j=r}^{\infty}(-1)^{j} \Delta^{j} \mu_{m+1}-(-1)^{r} \Delta^{r} \mu_{m+1} \\
& =\sum_{j=r+1}^{\infty}(-1)^{j} \Delta^{j} \mu_{m+1},
\end{aligned}
$$

which means that (12) is valid for $k=r+1$. Therefore, by mathematical induction, (12) is valid for all $k \in \mathbb{N}_{0}$. The proof of Theorem 10 is completed.

Proof of Theorem 11 By the definition of completely monotonic sequence, we only need to prove that

$$
(-1)^{k} \Delta^{k} \mu_{0} \geqq 0, \quad k \in \mathbb{N}_{0} .
$$

We first prove that

$$
(-1)^{k} \Delta^{k} \mu_{0} \geqq \sum_{j=k}^{\infty}(-1)^{j} \Delta^{j} \mu_{1}, \quad k \in \mathbb{N}_{0} .
$$

From the condition of Theorem 11, (22) is valid for $k=0$. 
Suppose that (22) is valid for $k=m$. Then we have

$$
\begin{aligned}
(-1)^{m+1} \Delta^{m+1} \mu_{0} & =(-1)^{m+1}\left(\Delta^{m} \mu_{1}-\Delta^{m} \mu_{0}\right) \\
& =(-1)^{m}\left(\Delta^{m} \mu_{0}-\Delta^{m} \mu_{1}\right) \\
& =(-1)^{m} \Delta^{m} \mu_{0}-(-1)^{m} \Delta^{m} \mu_{1} \\
& \geqq \sum_{j=m}^{\infty}(-1)^{j} \Delta^{j} \mu_{1}-(-1)^{m} \Delta^{m} \mu_{1} \\
& =\sum_{j=m+1}^{\infty}(-1)^{j} \Delta^{j} \mu_{1},
\end{aligned}
$$

which means that (22) is valid for $k=m+1$. Therefore, by mathematical induction, (22) is valid for all $k \in \mathbb{N}_{0}$.

Since

$$
\sum_{j=0}^{\infty}(-1)^{j} \Delta^{j} \mu_{1}
$$

is a convergent positive series, we know that

$$
\sum_{j=k}^{\infty}(-1)^{j} \Delta^{j} \mu_{1} \geqq 0, \quad k \in \mathbb{N}_{0} .
$$

From (22) and (24), we obtain that

$$
(-1)^{k} \Delta^{k} \mu_{0} \geqq 0, \quad k \in \mathbb{N}_{0} .
$$

The proof of Theorem 11 is completed.

Proof of Theorem 12 By Definition 2 and by setting $m=0$ in Theorem 9, we see that the condition is necessary. By Theorem 11, we know that the condition is sufficient. The proof of Theorem 12 is completed.

\section{Competing interests}

The authors declare that they have no competing interests.

\section{Authors' contributions}

All the authors contributed to the writing of the present article. They also read and approved the final manuscript.

\section{Author details}

'Department of Mathematics, Zhongyuan University of Technology, Zhengzhou, Henan 450007, People's Republic of China. ${ }^{2}$ Department of Mathematics and Statistics, University of Victoria, Victoria, British Columbia V8W 3R4, Canada.

${ }^{3}$ Department of Mathematics, Faculty of Arts and Sciences, Nevşehir University, Nevşehir, 50300, Turkey.

\section{Acknowledgements}

The authors thank the editor and the referees, one of whom brought our attention to the reference [6], for their valuable suggestions to improve the quality of this paper. The present investigation was supported, in part, by the Natural Science Foundation of Henan Province of China under Grant 112300410022. 


\section{References}

1. Widder, DV: The Laplace Transform. Princeton University Press, Princeton (1946)

2. Wimp, J: Sequence Transformations and Their Applications. Academic Press, New York (1981)

3. Lorch, L, Moser, L: A remark on completely monotonic sequences, with an application to summability. Can. Math. Bull. 6, 171-173 (1963)

4. Feller, W: An Introduction to Probability Theory and Its Applications, vol. 2. Wiley, New York (1966)

5. Kimberling, $\mathrm{CH}$ : A probabilistic interpretation of complete monotonicity. Aequ. Math. 10, 152-164 (1974)

6. Kimberling, CH: Exchangeable events and completely monotonic sequences. Rocky Mt. J. Math. 3, 565-574 (1973)

7. Kuk, AYC: A litter-based approach to risk assessment in developmental toxicity studies via a power family of completely monotone functions. J. R. Stat. Soc. C 53, 369-386 (2004)

8. Sandhya, E, Satheesh, S: On distribution functions with completely monotone derivative. Stat. Probab. Lett. 27, 127-129(1996)

9. Satheesh, S, Sandhya, E: Distributions with completely monotone probability sequences. Far East J. Theor. Stat. 1, 69-75 (1997)

10. Trimble, SY, Wells, J, Wright, FT: Superadditive functions and a statistical application. SIAM J. Math. Anal. 20, 1255-1259 (1989)

11. Day, WA: On monotonicity of the relaxation functions of viscoelastic materials. Proc. Camb. Philos. Soc. 67, 503-508 (1970)

12. Franosch, T, Voigtmann, T: Completely monotone solutions of the mode-coupling theory for mixtures. J. Stat. Phys. 109, 237-259 (2002)

13. Alzer, H, Batir, N: Monotonicity properties of the gamma function. Appl. Math. Lett. 20, 778-781 (2007)

14. Batir, N: On some properties of the gamma function. Expo. Math. 26, 187-196 (2008)

15. Guo, S: Logarithmically completely monotonic functions and applications. Appl. Math. Comput. 221, 169-176 (2013)

16. Guo, S: Some properties of completely monotonic sequences and related interpolation. Appl. Math. Comput. 219, 4958-4962 (2013)

17. Guo, S, Qi, F: A class of logarithmically completely monotonic functions associated with the gamma function. J. Comput. Appl. Math. 224, 127-132 (2009)

18. Guo, S, Qi, F, Srivastava, HM: A class of logarithmically completely monotonic functions related to the gamma function with applications. Integral Transforms Spec. Funct. 23, 557-566 (2012)

19. Guo, S, Qi, F, Srivastava, HM: Supplements to a class of logarithmically completely monotonic functions associated with the gamma function. Appl. Math. Comput. 197, 768-774 (2008)

20. Guo, S, Qi, F, Srivastava, HM: Necessary and sufficient conditions for two classes of functions to be logarithmically completely monotonic. Integral Transforms Spec. Funct. 18, 819-826 (2007)

21. Guo, S, Srivastava, HM: A certain function class related to the class of logarithmically completely monotonic functions. Math. Comput. Model. 49, 2073-2079 (2009)

22. Guo, S, Srivastava, HM: A class of logarithmically completely monotonic functions. Appl. Math. Lett. 21, 1134-1141 (2008)

23. Guo, S, Xu, J-G, Qi, F: Some exact constants for the approximation of the quantity in the Wallis' formula. J. Inequal. Appl. 2013, $67(2013)$

24. Qi, F, Guo, S, Guo, B-N: Complete monotonicity of some functions involving polygamma functions. J. Comput. Appl. Math. 233, 2149-2160 (2010)

25. Sevli, H, Batir, N: Complete monotonicity results for some functions involving the gamma and polygamma functions Math. Comput. Model. 53, 1771-1775 (2011)

26. Srivastava, HM, Guo, S, Qi, F: Some properties of a class of functions related to completely monotonic functions. Comput. Math. Appl. 64, 1649-1654 (2012)

27. Widder, DV: Necessary and sufficient conditions for the representation of a function as a Laplace integral. Trans. Am. Math. Soc. 33, 851-892 (1931)

28. Lorch, L, Newman, DJ: On the composition of completely monotonic functions, completely monotonic sequences and related questions. J. Lond. Math. Soc. 28, 31-45 (1983)

10.1186/1687-1847-2013-294

Cite this article as: Guo et al.: A certain class of completely monotonic sequences. Advances in Difference Equations 2013, 2013:294

\section{Submit your manuscript to a SpringerOpen ${ }^{0}$ journal and benefit from:}

- Convenient online submission

- Rigorous peer review

- Immediate publication on acceptance

- Open access: articles freely available online

- High visibility within the field

- Retaining the copyright to your article

Submit your next manuscript at $>$ springeropen.com 